\title{
A Fast and Accurate Position Sensitive Timing Detector for Charged Particles
}

\author{
D. Shapira, T. A. Lewis
}

\begin{abstract}
-
A position-sensitive timing detector suitable for detection of low-energy heavy ions is described. The device is based on the detection of secondary electrons emitted following ion impact on a surface. Using a combination of magnetic and electric fields these devices can deliver sub-nanosecond time definition and sub-millimeter position information for ion impact. When choosing a thin foil for the surface intercepting the heavy ion trajectory, multiple detectors can he used and reliable tracking of heavy ions with moderate energies $(\geq 1 \mathrm{MeV} / \mathrm{A})$ becomes feasible.
\end{abstract}

\section{INTRODUCTION}

In studies where particle detection and identification is required there is a prevailing need for charged particle detectors with the best available position and timing resolution. The use of particle tracking in magnetic fields for filtering reaction products is widespread in studies involving light particles at relativistic energies. Recently, tracking of more complex particles in large spectrographs and solenoids has been introduced in studies of heavy-ion induced reactions [1], [2], [3]. As numerous similar complex devices are being planned, the need for heavyion tracking will become more acute [4], [5], [6]. Many future studies are planned with radioactive beams where [2] impurities in the incident beam and some spread in phase space can be expected. In such situations, tracking of beam particles can help increase acceptance of the magnetic spectrograph or solenoids, and if accurate enough, it will provide better beam and/or species definition [7]. With present day devices, accurate and efficient tracking of heavy ions with energies below a few $\mathrm{MeV} / \mathrm{A}$ is virtually impossible. Excessive energy loss and multiple scattering of the particle in the first of a series of tracking detectors prevents accurate tracking. The detector that we report on in this study addresses some of the inherent difficulties.

Particularly suitable detectors for heavy particle tracking are those that require the least material for the detected ions to traverse. Some advances have been made in building thin gas-filled detectors, but even these need at least two foils, which must be strong enough to contain the detector's active gas [8], [9].

Detectors that use a single thin foil make for an optimal solution. Very thin scintillators have been used as transmission detectors, but they require elaborate light collection mechanisms and must still be thick enough to produce sufficient light output " [10]. A different solution involves detecting secondary electrons emitted from a thin foil following the passage of an ion. These few electrons emitted from the foil are multiplied in a micro-channel plate detector equipped with a position-sensing anode. A large fraction of secondary electron emission occurs

D. Shapira is a member of the Physics Division and T. A. Lewis is a member of the Engineering Science and Technology Division, Oak Ridge National Laboratory, Oak Ridge, TN 37831, USA.

Oak Ridge National Laboratory is managed by UT-Battelle, LLC under contract DE-AC05-000R22725 with the U.S. Department of Energy. at the surface, and in principle, this method should work with the thinnest possible foils.

Both detection methods mentioned above have been used successfully in applications requiring good timing, but have found limited use as position-sensitive detectors. This report presents a design of this detector type, which is thin enough to allow for heavy-ion tracking with excellent position resolution, good timing, and the ability to sustain high counting rates.

\section{The Detector}

\section{A. Detector Design and Principle of Operation}

Detectors in which the ions need to traverse only a single foil in order to determine the time and position of the passing ion have been in use for some time.

Fig. 1 shows the schematic layout of such a detector, designed to get timing and position of ion impact on a thin foil. Secondary electrons are emitted from the foil at the location of the ion impact, and are accelerated toward a fast electron multiplier such as a micro-channel plate detector (MCP). This and other similar arrangements provide an accurate time stamp for the ion's impact on the foil and have been used routinely in fast timing applications [11], [ 12], [ 13] with great success. Electron ejection from the foil is confined to the position where the passing ions hit the foil. In principle, the signals from these electrons that were accelerated toward the MCP detector should also be localized. Therefore, if the MCP detector's anode is replaced by a position-sensing plane [14], [15], this detector could also record the position of electron impact on the detector surface and yield, indirectly, information on the position where the passing ion hit the foil. Reported position resolution for several such detectors in use, where foil-to-MCP distances are at least a few centimeters, are no better than $2 \mathrm{~mm}$ (FWHM) [16], [17], [18]. Reference [19], which analyzes the performance of this genre of detectors, identifies electron transport from the foil to the detector as the main obstacle to attaining sub-millimeter definition of the heavy-ion impact position on the foil. The main cause of straying of the secondary electron trajectory from a straight path leading from foil to the detector is due to their initial lateral velocity. The impact of this lateral component can be minimized by rapid acceleration of the secondary electrons toward the detector [ 16], [ 18]. It has also been recognized that a magnetic field parallel to the direction of desired propagation will cause the electron to move in a tight spiral in its trek from foil to microchannel plate detector surface [20]. In Ref. [20], low magnetic fields were applied and accurate localization was based on the number of full turns executed by the electrons spiraling along the central trajectory. The predicted oscillatory behavior of position resolution on magnetic-field strength did not not show in their data, perhaps because the emitted electron spectrum poss- 


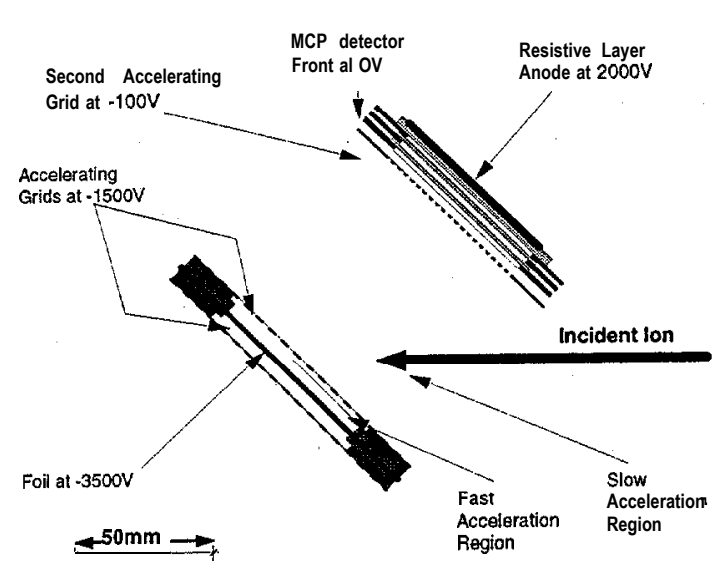

Fig. 1. A schematic diagram showing the Postion-sensitive timing detector setup. The foil to accelerating grids distance is approximately $3 \mathrm{~mm}$, microchannel and foil surfaces are $45 \mathrm{~mm}$ in diameter cach and $45 \mathrm{~mm}$ apart.

eses a countinuous spread of energy and emission angle. It is evident, however, from the discussions presented in Ref. [19], that optimal preservation of the information on the points of origin during electron transport from foil to micro-channel plate detector surface will require a combination of magnetic containment and electrostatic acceleration. Applying a strong magnetic field in the direction of desired electron motion should bring about better correspondence between the location where the electron was generated and where it reaches the detector.

In our design we follow the practice used in electron spectrometers [21], [22], [23]. The so-called beam parallelizer is used to collect electrons of a few $\mathrm{eV}$ energy diverging from a source and turn them into a parallel beam. We applied a strong, but not necessarily uniform, magnetic field and kept electron acceleration to a minimum. We allowed enough acceleration'to keep the time spread in electron transit from foil to MCP detectors low. Electron motion under the combination of small electrostatic acceleration and an inhomogeneous magnetic field is described in the articles discussing magnetic beam parallelizers [23]. In this device, lateral momentum is slowly converted to longitudinal momentum. As long as the magnetic field is strong and electrostatic acceleration moderate, the electrons will move along the magnetic field line in spirals.

\section{B. Detector Construction}

A schematic diagram showing the essential parts of the detector assembly is shown in Fig. 2. In several aspects this detector is similar to the detector shown in Fig. 1. It has the same foil with accelerating grids close by and the micro-channel plate detector facing it. The whole assembly is tilted at $45^{\prime \prime}$ or $30^{\circ}$ to the beam axis and the detector is removed enough from the foil so that the beam can pass through unimpeded. This design is different in the voltages applied to the foils and grids and the presence of two permanent disk magnets. The disks are Sm magnets approximately 2 " in diameter with thicknesses of 1 " and $1 / 2$ " each. In the arrangement shown here, they generate a fairly uniform magnetic-field strength between the foil and detector planes of approximately $1 \mathrm{~K}$-gauss. This detector configuration provided very little image magnification, (about 110\%) for the pattern

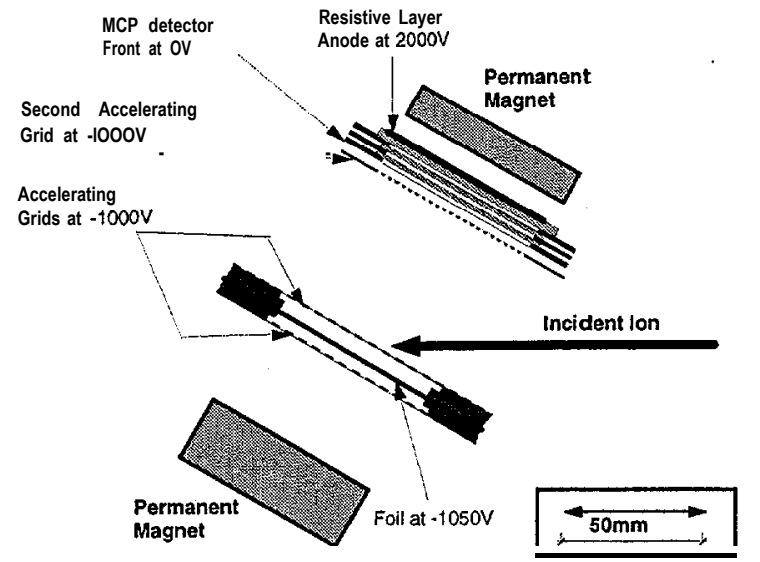

Fig. 2. A schematic diagram showing the modified position-sensitive timing detector setup. The dimensions are similar to those which appear in Fig. 1.

produced at the foil. The combination of these magnets and their relative placement can be used to provide varying configurations of magnetic fields. Some of the tests shown in this work were done with the magnets placed in such a way that the field produced was strong and nearly homogeneous. In other tests, the magnet placed behind the MCP detector was removed, allowing the magnetic-field lines to diverge resulting in substantial magnification. Still in other tests, the magnet placed behind the foil was removed allowing the magnetic field lines to to converge on the MCP detector resulting in substantial de-magnification.

\section{Detector Performance}

\section{A. Position Resolution}

The setup used for testing the performance of the detection system is similar to the one described in Ref. [19] and is shown here in Fig. 3. In the tests reported here, the mask shown in Fig. 3 is placed close to the detector foil (approx 2" away) in order to minimize any spreading of the image due to multiple scattering at the foil. The signal processing diagram is also shown in Fig. 3. Timing signals from the MCP, picked up from swings in the final biasing electrode, are measured in coincidence with signals from the scintillation detectors. This ensures processing of signals that cleared the mask. The position spectra, a twodimensional pattern as well as a projected slice of the spectrum, are shown in Fig. 4. The performance of the detector is quite good. Even the yield of ion groups delineated by the smallest holes measuring 0.5 and $0.3 \mathrm{~mm}$ stand out clearly. The position resolution in the spectrum is slightly below $0.4 \mathrm{~mm}$, and at this level, multiple scattering at the foil as well as signal processing by the detector could play a role.

In order to check the ultimate position definition attainable with this device, image definition has to occur as close to the foil as possible in order to keep effects of multiple scattering at a bare minimum. To further test the position resolution of this detector, we placed a mask with a few strips and wires close to the foil on the downstream side. The mask is shown in Fig. 5 and the resulting pattern, defined by coincidences between this detector and the scintillation detector, is shown in Fig. 6. Outline of the images of the two IO-mil wires (1/4-mm) placed on this mask are evident. 


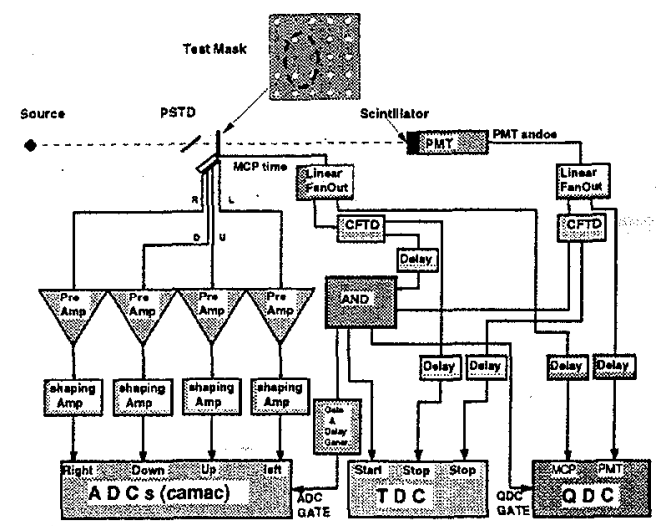

Fig. 3. Adiagram showing our first test setup- position definition is with a mass placed close to the foil. The mask is the same used in a previous publication [19].

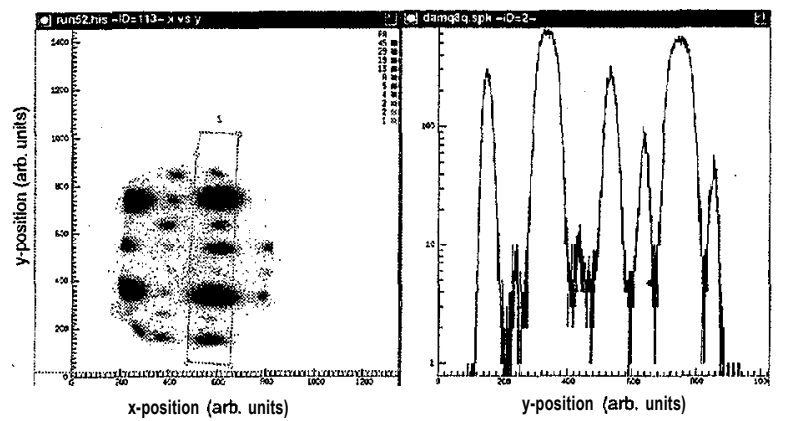

Fig. 4. A two-dimensionial histogram of hit pattern of electrons on the MCP detector and a projection of a slice along one of the axes. The slit pattern being projected is of eight circular holes with the following diameters (listed top to bottom): $1.0,4.0,1.0,2.0,0.5,4.0,0.25$, and $2.0 \mathrm{~mm}$.

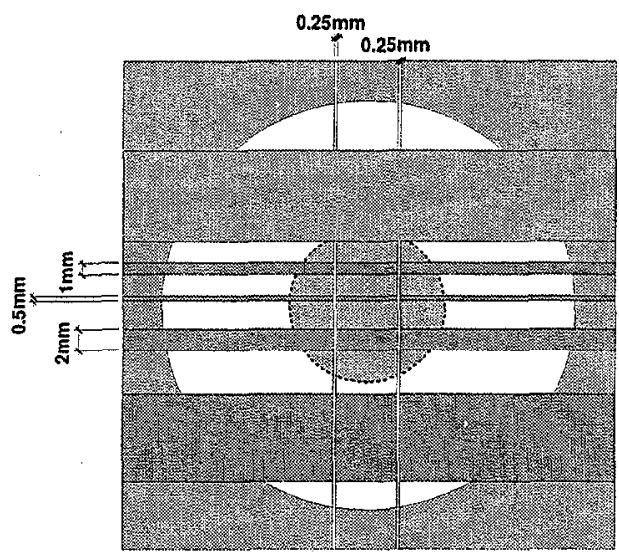

Fig. 5. Details of the mask placed next to the foil in the final test of detector position resolution.

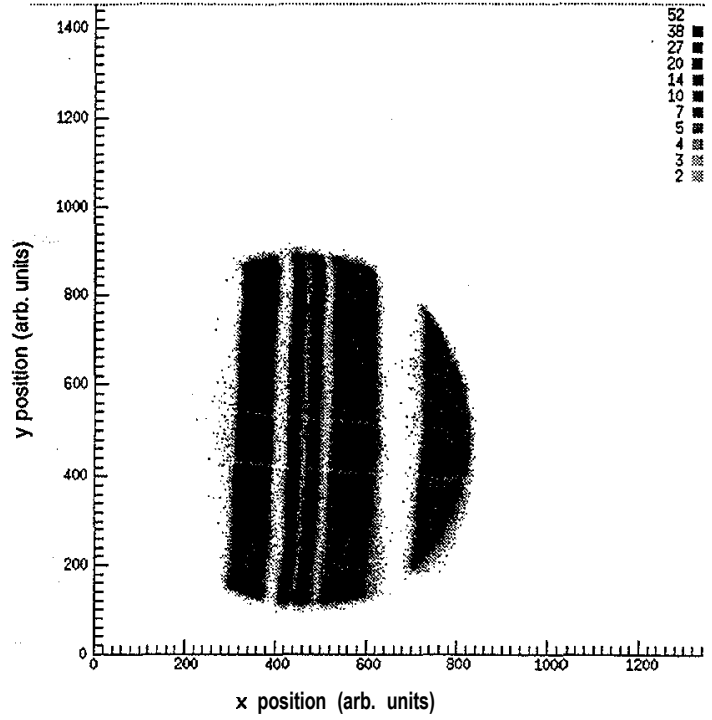

Fig. 6. A two-dimensional histogram ofelectron hits on the MCP detector. Only $50 \mathrm{~V}$ were used to accelerate the electron produced near the foil.

\section{CONClusions And Further Improvements}

As demonstrated here, the detector can render superior position resolution while retaining much of the timing resolution and its counting rate capabilities. Our tests show that the bandwidth of the position decoding hardware and electronics may be the greatest bottle neck. The company that supplied the resistive layer [24] used in the position decoding also has resistive layer anodes with lower resistivity that would stand higher count rates. By using a high quality wide-band charge-sensitive preamplifier in conjunction with the low-resistivity layer, one may be able to acquire data at the maximum rate allowed by this device and still get good position decoding.

As mentioned in Section 2.2, one intriguing aspect of this detector is the fact that it can be used to magnify or demagnify the image on the foil. Fig. 7 shows the position spectrum obtained with the same mask shown in Fig. 7 but with one of the magnets removed (the one behind the micro-channel plates). The resulting magnification is apparent. Fig. 8 shows a one-dimensional projection in which the two troughs generated by the $0.25-\mathrm{mm}$ wires are apparent. The smaller structure seen in this figure as well as in the two-dimensional display (Fig. 7) is due to the accelerating grid, which was made from a wire mesh of $0.01 \mathrm{~mm}$ wires. Fig. 9 shows the image of a mesh structure that was $11 \mathrm{x}$ $7 \mathrm{~cm}$ overall and made of $1 \mathrm{~mm}$ diameter wires on $1 \mathrm{~cm}$ centerto-center spacing. The image demagnification ratio is 3 to 1 .

\section{ACKNOWLEDGEMENTS}

Research at the Oak Ridge National Laboratory is supported by the U.S. Department of Energy under contract DE-AC-0500OR22725 with UT-Battelle, LLC.

\section{REFERENCES} (1993), 1.56 . 


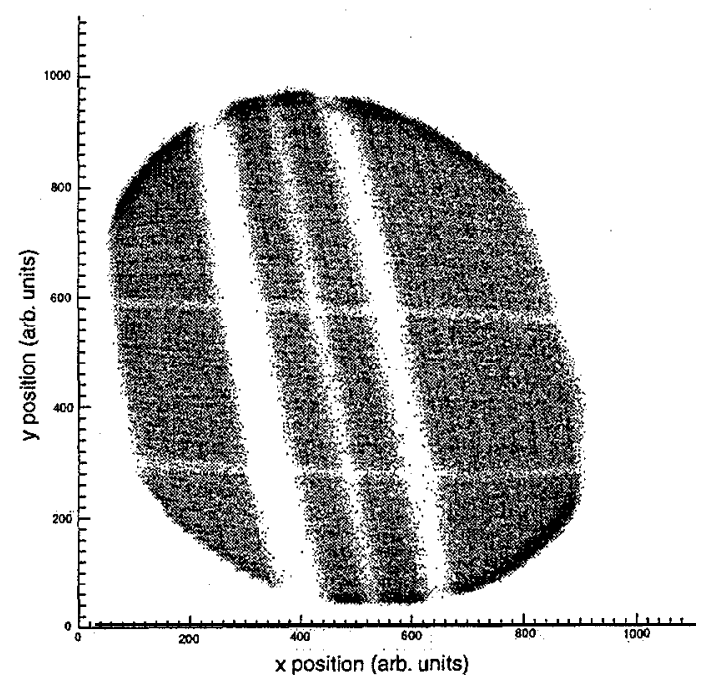

Fig. 7. A two-dimensional histogram of electron hits on the MCP detector with magnetic-field strength decreasing between foil and MCP.

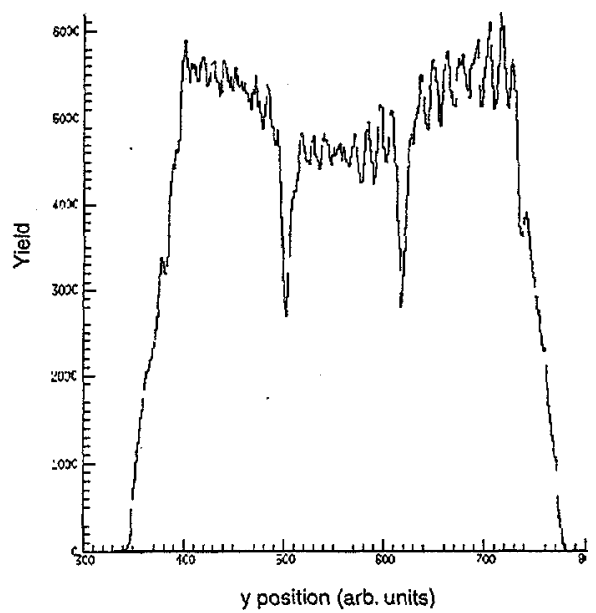

Fig. 8. A projection along the direction of the two thin wires seen in the image of Fig. 9.

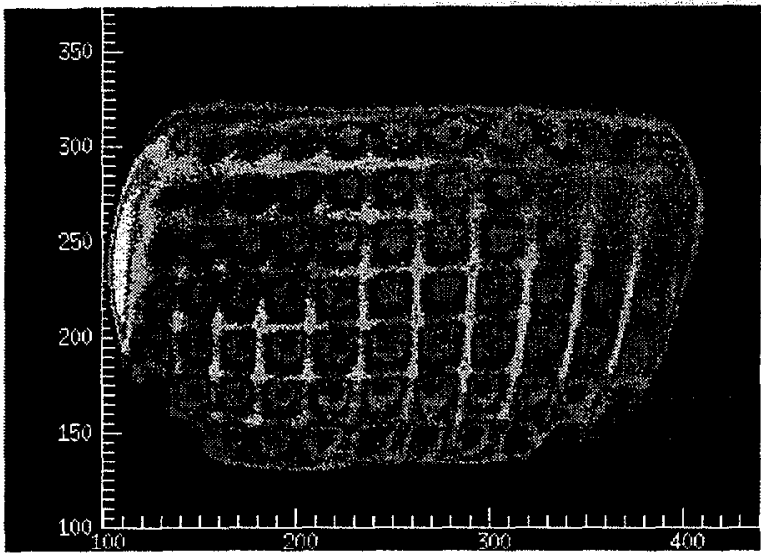

Fig. $9.7 \times 12 \mathrm{~cm}$ mesh of $1 \mathrm{~mm}$ diameter wires on $1 \mathrm{~cm}$ center-to-center spacing $3 \mathrm{x}$ demagnification.

[2] J. J. Kolata. "First Results from the TWINSOL RNB Facility," Revista Mexicana de Fisica Suplemento 44, 92 and Nuclear Instr. and Meth. in Physics Research B 40/41 (1989) 503.

[3] F. D. Becchetti, "Low-Energy Radioactive Beam Experminents Using the UM-UND Solenoid RNB Apparatus at the UND Tandem," Proceedings of Fourteenth Conference on Application of Accelerators in Research and Industry, AIP Conference Proceedings 392, p. 369.

[4] J. M. D'Auria, "A recoil mass separator for radiative capture reactions," p. 373 in Proceedings of the Workshop on the Experimental Equipment for an Advanced ISOL Facility, Ed. I. Y. Lee, LBNL42138, July 1998.

[5] H. Savajols, "A Variable Mode High Acceptance Spectrometer," p. 407 in Proceedings of the Workshop on the Experimental Equipment for an Advanced ISOL Facility, Ed. I. Y. Lee, LBNL-42138, July 1998.

[6] F. Scarlasara, "PRISMA: A large acceptance magnetic spectrograph for Legnaro National Lab., p. 429 in Proceedings of the Workshop on the Experimental Equipment for an Advanced ISOL Facility, Ed. I. Y Lee, LBNL-42138, July 1998.

[7] K. E. Zyromski, W. Loveland, G. A. Souliotis, D. J. Morrisey, C. F. Powell, 0. Batenkov, K. Aleklett, R. Yanez, I. Forsberg, M. Sanchez-Vega, J. R. Dunn, and B. G. Glagola, Phys. Rev. C 55 (1997) R562.

[8] K. E. Rehm and F. L. H. Wolfs, Nucl. Instr. and Meth. A 273 (1988) 262.

[9] A. Breskin, R. Chechik, Z. Fraenkel, I? Jacobs, I. Tseruya and N. Zwanhg, Nucl. Instr. and Meth. 221(1984) 363.

[10] W. Muller, F. J. Hartmann, J. Eades, R. S. Hayano, B. Ketzer and F. E. Maas, Nucl. Instr. and Meth. A 349 (1994) 307.

[11] G. Gabor, W. Schimmerling, D. Greiner, F. Bleser and P. Lindstrom, Nucl. Instr. and Meth. 130 (1975) 65.

[12] W. Starzecki, A. M. Stefanini, S. Lunardi and C. Signorini, Nucl. Instr. and Meth 193 (1982) 499.

[13] E. C. Pollaco et. al., Nucl. Instr. and Meth. 225 (1984) 51.

[14] J. L. Culhane, Nucl. Instr. und Meth. A 310 (1991) 1.

[15] C. H. W. Sigmund, M. Lampton, R. Raffant and W. Merrick, Nucl Instr. and Meth. A 310 (1991) 311.

[16] A. N. James, T. P. Morrison, K. L. Ying, K. A. Connell, H. G. Price and J. Simpson, Nucl. Instr. and Meth. A 267 (I 988) 144.

[17] R. A. Cunningham, private communication.

[18] W. Gellently, private communication.

[19] D. Shapira, T. A. Lewis, L. D. Hulett and Z. Ciao, Nucl. Instr. and Meth. 449 (2000) 396-407.

[20] 0. H. Odland, W. Mittig, A. Lepine-Szily, G. Fremont. M. Chat-trier, M. MacCormick, J. M. Casndjian, Nucl. Instr. and Meth. A 378 (1996) 149 .

[21] T. Hsu and J. L. Hirshfield, Rev. Sci. Instrum. 47 (1976) 236.

[22] G. Beamson, H. Q. Porter and D. W. Turner, J. Phys. E: Sci. Instrum. 13 (1980) 64

[23] P. Kmit and F. H. Read, J. Phys. E: Sci. Instrum. 16 (1983) 313.

[24] Quantar Technology Inc., 200 Washington Street, Santa Cruz, CA 95060-4976. 